acta medica

\title{
Ear Nose and Throat Involvement in AAV
}

\section{Assoc. Prof. Serdar Özer, MD}

ORCID: 0000-0003-1669-8020

Note:

This manuscript has been peer-reviewed by Assoc. Prof. Dr. Riza Önder Günaydın before acceptance.
Hacettepe University Faculty of Medicine, Department of Ear Nose and Throat, Ankara
NCA associated vasculitis (AAV) is typically characterized by necrotizing, granulomatous inflammation of the upper and lower airways, focal or proliferative glomerulonephritis, and other vasculitis-related conditions that may accompany them. While Ear Nose and Throat (ENT) involvement is not a typical finding in microscopic polyanginitis (PA), it is among the typical findings in Granulomatous PA and Eosinophilic Granulomatous PA and appears as the first and sometimes the only involvement of the cases. AAV cases require a multidisciplinary approach.

As ENT specialists, although we mostly see sinonasal region involvement in cases with AAV, ear, larynx, salivary gland and oral cavity involvements could also be seen. In cases, findings such as allergic rhinitis, nasal polyposis, chronic sinusitis, otitis media with effusion, sensorineural hearing loss, facial paralysis, tinnitus, dizziness, ulcerative stomatitis and sialadenitis are frequently encountered in the practice of ENT specialists and cannot be predicted in the first evaluation with AAV. Apart from these, there may be situations like nasal septal perforation, saddle nose deformity, laryngotracheal stenosis, oro-antral fistula and osteonecrosis in which we definitely seek for underlying pathologies and go for further evaluation.

Examination and evaluation of the nose and paranasal sinuses is important because it is a region where initial involvement can be seen and active disease evaluation is made. Chronic sinusitis and nasal polyposis are the most common findings. Idiopathic nasal septal perforation is also one of the most common findings and hemorrhagic fragile nasal mucosa is one of the most pathognomonic examination findings. Otological findings generally develop due to eustachian tube dysfunction and otitis media with effusion is seen in most of the cases. Ossicular system destruction and mastoiditis due to granulomatous involvement of the middle ear can rarely be seen. In the presence of inner ear involvement, dizziness, facial paralysis and progressive sensorineural hearing loss can be seen. Laryngeal involvement usually manifests itself with laryngotracheal ulcers and can cause complications such as subglottic stenosis in the late period.

As ENT specialists, we play a key role in the diagnosis of ANCA-related cases and in the planning of their treatment by evaluating the disease activity. We can only make this contribution by being aware of these diseases and by following the patients closely. 